\title{
Multiagent Coordination Exploiting System Symmetries
}

\author{
Bill Goodwine and Panos Antsaklis
}

\begin{abstract}
This paper presents results related to the multiagent formation control problem. Symmetries in the system are exploited to simplify the stability analysis and control synthesis problem for symmetric systems. The type of symmetry considered is a discrete symmetry where individual agents are either identical or have dynamics that are diffeomorphically related. These results are applicable to both distributed as well as non-distributed coordination methods and are demonstrated with simulation results for systems taken from the literature.
\end{abstract}

\section{INTRODUCTION}

Formation control for multiple mobile robotic systems has a long history, with the main focus being on the use of potential functions for coordination (see for example [15], [3], [13] and the citations therein). The use of potential functions has an obvious appeal in that they facilitate stability analyses using Lyapunov functions. The drawbacks are wellknown also, which include among other things, the existence of multiple local minima in complex environments, the fact that realistic potential functions representing the realities of sensor ranges introduce mathematical limitations on the potential functions which complicate and limit the stability analysis etc. As observed in [12], many of the prior efforts have assumed specific dynamics with the correct observation that they probably generalize; however, our approach in this paper is intended to be much more general. Perhaps the work closest to this present work be that of [12] wherein a control Lyapunov function is assumed to exist for each agent, from which formation functions and bounds on formation speed can be derived to ensure stability. The added benefit of the results in this paper is that our formulation provides the type of cases and underlying structure for systems to which the results in [12] will apply. Furthermore, our results here apply to a broader class of systems, such as fully distributed ones, to which the results in [12] do not necessarily apply.

The main contributions of this paper are 1) a nonlinear extension of the results in [1], [14] which is a more straightforward representation of system symmetries than our previous work; 2) the presentation of a unified theoretical framework that seems to be underlying many of the formation control algorithms in the literature; and, 3) a general stability theorem that is applicable to such systems regardless of the number of components. These results will allow a control design engineer to focus the analysis on a smaller, more tractable system with a guarantee that stability conclusions

Bill Goodwine is with the Department of Aerospace \& Mechanical Engineering, University of Notre Dame, Notre Dame, Indiana 46556 USA goodwinedcontrols. ame.nd.edu

Panos Antsaklis is with the Department of Electrical Engineering at the University of Notre Dame, Notre Dame, Indiana 46556 USA pantsakl@ind.edu

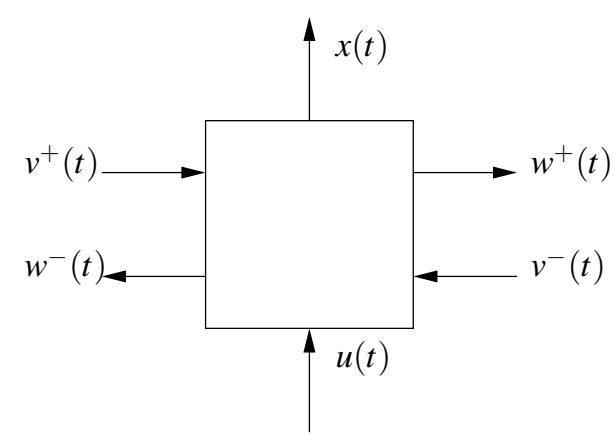

Fig. 1. System building block in one spatial dimension.

will hold for a much larger system. It is emphasized that the two examples presented in Section IV were originally published with analyses that were not limited to the number of components - the contribution here being making the underlying theoretical formulation explicit.

This paper essentially extends the previous work of one of the authors related to the properties of symmetric systems [9], [7], [8], [11], [10] to consider nonlinear system stability. The main application in this paper is formation control of multiple robotic agents, and it is applied to two cases, one of which is decentralized and the other of which is not.

The previous work cited considers system symmetries that are defined by a group action on the configuration manifold for a distributed system that was induced by the action of a permutation group. The main drawback of such an approach is that, in the general case, identifying such symmetries can be problematic. However, in the case of most engineering and robotics systems, where the individual robots are the components that are symmetric, symmetry identification is much less of a problem. Rather than using this prior approach, this paper will introduce a more straightforward approach which is a nonlinear extension of the approach used in [1], [14]. However, it is emphasized that the prior approaches [6], [9], [7], [8], [11], [10], [5] offer a general approach to the problem that can be used in cases more general than the ones addressed here.

\section{SyMmETRIC SYSTEMS}

The approach is to extend the basic system component from [1] to the nonlinear case. The "basic building block" in one spatial dimension is illustrated in Figure 1. The outputs from the component are $w^{-}(t)$ and $w^{+}(t)$, and the inputs are $u, v^{-}(t)$ and $v^{+}(t)$. The signals $v^{ \pm}$represent the effects of the coupling with the other components and $u$ are the usual control inputs which need to be designed for stability, performance, robustness, etc. 
We wish to express component-by-component, the usual dynamics of a nonlinear control system expressed by

$$
\dot{x}=f(x)+\sum_{j=1}^{m} g_{j}(x) u_{j},
$$

where $x \in \mathbb{R}^{n}$ and the vector fields $f(x), g_{j}(x) \in T \mathbb{R}^{n}$. In the general case, the vector fields $f$ and $g_{j}$ depend on the coupling with the other components, so the equations governing the dynamics of this component are given by

$$
\begin{aligned}
\dot{x}_{i}(t)= & f_{i}\left(x_{i}(t), v_{i}^{+}(t), v_{i}^{-}(t)\right) \\
& +\sum_{j=1}^{m} g_{j, i}\left(x_{i}(t), v_{i}^{+}(t), v_{i}^{-}(t)\right) u_{j}(t) \\
w_{i}^{-}(t)= & \hat{f}_{i}^{-}\left(x_{i}(t), v_{i}^{+}(t), v_{i}^{-}(t)\right), \\
w_{i}^{+}(t)= & \hat{f}_{i}^{+}\left(x_{i}(t), v_{i}^{+}(t), v_{i}^{-}(t)\right),
\end{aligned}
$$

which is the direct nonlinear extension of the system developed in [1]. For a system of interconnected components where the incoming signals, $v^{ \pm}(t)$ are from the outgoing signals from the component's neighbors, the entire system is coupled because the $w^{ \pm}(t)$ signals depend on the component's input signals, $v^{ \pm}(t)$. The class of the types of coupling that could be represented by this formulation is very broad and could include, for example, when there is a physical joining of agents, as with reconfigurable, modular robots.

For mobile robots where there is no physical contact between the robots and the topology of the structure of the system is such that a component only receives a limited number of inputs (from its nearest neighbors, for example), the nature of the coupling between the robots can be expressed in a much simpler manner. In particular, it is only through the control inputs that the output from the other components affects the dynamics of an agent, which is expressed by

$$
\begin{aligned}
\dot{x}_{i}(t) & =f_{i}\left(x_{i}(t)\right)+\sum_{j=1}^{m} g_{j, i}\left(x_{i}(t)\right) u_{j}(t) \\
w_{i}^{-}(t) & =\hat{f}_{i}^{-}\left(x_{i}(t)\right) \\
w_{i}^{+}(t) & =\hat{f}_{i}^{+}\left(x_{i}(t)\right)
\end{aligned}
$$

where writing

$$
u_{j, i}(t)=u_{j, i}\left(t, x_{i}(t), v_{i}^{+}(t), v_{i}^{-}(t)\right)
$$

makes this explicit. If the control is determined only by static state feedback, then $u_{j, i}(t)=u_{j, i}\left(x_{i}(t), v_{i}^{+}(t), v_{i}^{-}(t)\right)$.

In a manner analogous to the approach in [1], we can build up a system with $N$ components by requiring periodic interconnections in one dimension, i.e.,

$$
v_{i+1}^{+}(t)=w_{i}^{+}(t), \quad v_{i-1}^{-}(t)=w_{i}^{-}(t),
$$

where all mathematical operations on indices are $\bmod (N)$. In this case, feedback can be expressed in terms of the outputs from the neighbors and Equation 2 can be written as

$$
u_{j, i}=u_{j, i}\left(t, x_{i}(t), w_{i-1}^{+}\left(x_{i-1}(t)\right), w_{i+1}^{-}\left(x_{i+1}(t)\right)\right.
$$

or in the case of feedback only,

$$
u_{j, i}=u_{j, i}\left(x_{i}(t), w_{i-1}^{+}\left(x_{i-1}(t)\right), w_{i+1}^{-}\left(x_{i+1}(t)\right) .\right.
$$

The simplest type of symmetric system would be when all the components are identical with periodic interconnections, and example of which would be a fleet of autonomous robots that are in a formation where each robot communicates in an identical manner to its two nearest neighbors.

Definition 1 Consider a system of the form of Equation 1. This system is a symmetric system spatially connected in one dimension if it has periodic interconnections and

$$
\begin{aligned}
f_{i}(x) & =f_{k}(x), & g_{j, i}(x) & =g_{j, k}(x), \\
\hat{f}_{i}^{-}(x) & =\hat{f}_{k}^{-}(x), & \hat{f}_{i}^{+}(x) & =\hat{f}_{k}^{+}(x)
\end{aligned}
$$

for $x \in \mathbb{R}^{n}$, for all $i, k=1, \ldots, N$ and for each $j=1, \ldots, m$. A system under state feedback is a feedback symmetric system if it is a symmetric system and

$$
u_{j, i}\left(x_{1}, w_{i-1}^{+}\left(x_{2}\right), w_{i+1}^{-}\left(x_{3}\right)\right)=u_{j, k}\left(x_{1}, w_{k-1}^{+}\left(x_{2}\right), w_{k+1}^{-}\left(x_{3}\right)\right)
$$

for $\left(x_{1}, x_{2}, x_{3}\right) \in \mathbb{R}^{n} \times \mathbb{R}^{n} \times \mathbb{R}^{n}$, for all $i, k=1, \ldots, N$ and for each $j=1, \ldots, m$.

To allow more general topologies than connections in one spatial dimension, define the index sets $\mathcal{V}_{i}$ and $\mathcal{W}_{i}$ which index multiple inputs and outputs respectively for component $i$. The outputs from component $i$ are denoted by $w_{i}^{l}(t), l \in \mathcal{W}_{i}$ and the inputs are denoted by $v_{i}^{l}(t), l \in \mathcal{V}_{i}$.

The dynamics of a component are give by

$$
\begin{aligned}
& \dot{x}_{i}(t)=f_{i}\left(x_{i}(t)\right)+\sum_{j=1}^{m} g_{j, i}\left(x_{i}(t)\right) u_{j}(t) \\
& w_{i}^{l}(t)=\hat{f}_{i}^{l}\left(x_{i}(t)\right), \quad \forall l \in \mathcal{W}_{i}
\end{aligned}
$$

with

$$
u_{j, i}(t)=u_{j, i}\left(t, x_{i}(t) v_{i}^{l_{1}}(t), v_{i}^{l_{2}}(t), v_{i}^{l_{3}}(t), \ldots\right)
$$

where $l_{1}, l_{2}, \ldots \in \mathcal{V}_{i}$ and in the case of state feedback,

$$
u_{j, i}(t)=u_{j, i}\left(x_{i}(t) v_{i}^{l_{1}}(t), v_{i}^{l_{2}}(t), v_{i}^{l_{3}}(t), \ldots\right) .
$$

Periodic interconnections are defined in a manner similar to the case of one spatial dimension. In particular, if there exist orderings of each of the sets $\mathcal{V}_{i}$ and $\mathcal{W}_{i}$ and

$$
v_{i}^{j}(t)=w_{j}^{i}(t) \quad \forall j \in \mathcal{V}_{i}, \forall i \in \mathcal{W}_{j}
$$

then the system has periodic interconnections, which requires that $\mathcal{V}_{i}$ and $\mathcal{W}_{i}$ have the same size. For periodic interconnections, the input under feedback can be written as

$$
u_{j, i}\left(t, x_{i}(t), w_{i}^{l_{1}}\left(x_{l_{1}}(t)\right), w_{i}^{l_{2}}\left(x_{l_{2}}(t)\right), w_{i}^{l_{3}}\left(x_{l_{3}}(t)\right), \ldots\right)
$$

for $l_{1}, l_{2}, \ldots, \in \mathcal{W}_{i}$ or in the case of state feedback only

$$
u_{j, i}(t)=u_{j, i}\left(x_{i}(t), w_{i}^{l_{1}}\left(x_{l_{1}}(t)\right), w_{i}^{l_{2}}\left(x_{l_{2}}(t)\right), w_{i}^{l_{3}}\left(x_{l_{3}}(t)\right), \ldots\right) .
$$

The following example illustrates this type of scenario.

Example 1 A recurring example in this paper will be planar agents with second order dynamics used in [13]. Each robot 


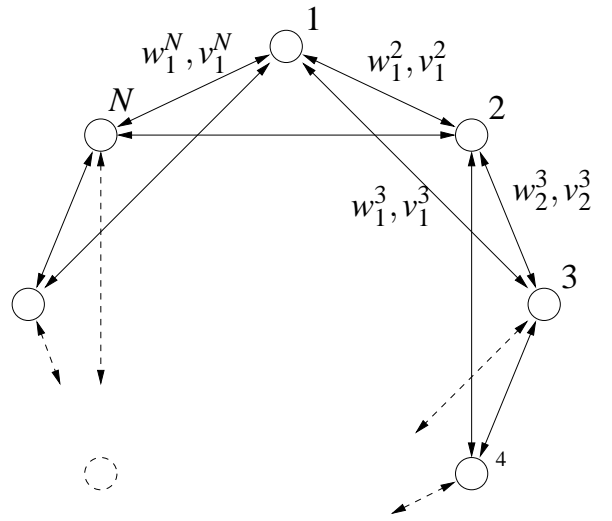

Fig. 2. System topology for Example 1.

has a location and velocity in $\mathbb{R}^{2}$, with equations of motion for the $i$ th robot given by

$$
\frac{d}{d t}\left[\begin{array}{c}
x_{i} \\
\dot{x}_{i} \\
y_{i} \\
\dot{y}_{i}
\end{array}\right]=\left[\begin{array}{c}
\dot{x}_{i} \\
0 \\
\dot{y}_{i} \\
0
\end{array}\right]+\left[\begin{array}{c}
0 \\
1 \\
0 \\
0
\end{array}\right] u_{1, i}+\left[\begin{array}{l}
0 \\
0 \\
0 \\
1
\end{array}\right] u_{2, i}
$$

The goal formation is a regular $N$-polygon and each robot communicates with its two neighbors and the next two agents, as is illustrated in Figure 2. In Figure 2, each node in the graph is a component similar to that illustrated in Figure 1; however, in this case each component has four outputs, each of which is simply its position and similarlyeach node has four inputs which are the outputs of the connected components, i.e.,

$$
w_{i}^{j}=\left[\begin{array}{l}
x_{i} \\
y_{i}
\end{array}\right], \quad v_{i}^{j}=\left[\begin{array}{l}
x_{j} \\
y_{j}
\end{array}\right]
$$

where $j \in\{i-2, i-i, i+1, i+2\}$. In the figure, each arrow represents both an input and output.

Let the desired formation distance between components $i$ and $j$ be

$$
d_{i j}= \begin{cases}1, & |i-j|=1 \\ \frac{\sin \left(\frac{2 \pi}{N}\right)}{\sin \left(\frac{\pi}{N}\right)}, & |i-j|=2\end{cases}
$$

which are the distances between the corresponding vertices for a regular $N$-polygon with unit side length.

Define the inputs to be as in [13] by

$$
\left[\begin{array}{c}
u_{1, i} \\
u_{2, i}
\end{array}\right]=-\sum_{j}\left[\frac{\frac{2\left(\sqrt{\left(x_{i}-x_{j}\right)^{2}+\left(y_{i}-y_{j}\right)^{2}}-d_{i j}\right)\left(x_{i}-x_{j}\right)}{\sqrt{\left(x_{i}-x_{j}\right)^{2}+\left(y_{i}-y_{j}\right)^{2}}}}{2\left(\sqrt{\left(x_{i}-x_{j}\right)^{2}+\left(y_{i}-y_{j}\right)^{2}}-d_{i j}\right)\left(y_{i}-y_{j}\right)}-k_{d}\left[\begin{array}{c}
\dot{x}_{i} \\
\dot{y}_{i}
\end{array}\right]\right.
$$

where $k_{d}$ is a positve constant damping gain and $j \in$ $\{i-2, i-1, i+1, i+2\}$.

This is a feedback symmetric system. In detail, by construction the dynamics for each agent are the same, i.e., $f_{i}(x)=f_{j}(x)$ and $g_{j, i}=g_{j, k}$. The index sets are

$$
\mathcal{V}_{i}=\mathcal{W}_{i}=\{i-2, i-1, i+1, i+2\}
$$

Each agent outputs its position, so $w_{i}^{l}(x)=\hat{f}_{i}^{l}(x)=\left[\begin{array}{ll}x_{i} & y_{i}\end{array}\right]^{T}$. Finally, since the inputs are a function of the state of component $i$ and the components to which it is connected

$$
\left[\begin{array}{l}
u_{1, i} \\
u_{2, i}
\end{array}\right]=\left[\begin{array}{l}
u_{1, i}\left(x_{i}, v_{i}^{i-2}, v_{i}^{i-1}, v_{i}^{i+1}, v_{i}^{i+2}\right) \\
u_{2, i}\left(x_{i}, v_{i}^{i-2}, v_{i}^{i-1}, v_{i}^{i+1}, v_{i}^{i+2}\right)
\end{array}\right] .
$$

Thus, the system satisfies all the elements of the definition of a symmetric feedback system.

Finally, we want to "build up" a system.

Definition 2 Given a a symmetric system spatially connected in one dimension with $N$ components we will call the system with $N+1$ components equivalent to it if

$$
\begin{aligned}
f_{i}(x) & =f_{k}(x), & g_{j, i}(x) & =g_{j, k}(x), \\
\hat{f}_{i}^{-}(x) & =\hat{f}_{k}^{-}(x), & \hat{f}_{i}^{+}(x) & =\hat{f}_{k}^{+}(x)
\end{aligned}
$$

for $x \in \mathbb{R}^{n}$, for all $k=1, \ldots, N$ and for all $i=1, \ldots, N+1$ and for each $j=1, \ldots, m$. The $N+1$ system is feedback equivalent to the $N$ system if

$$
u_{j, i}\left(x_{1}, w_{i-1}^{+}\left(x_{2}\right), w_{i+1}^{-} x_{3}\right)=u_{j, k}\left(x_{1}, w_{k-1}^{+}\left(x_{2}\right), w_{k+1}^{-}\left(x_{3}\right)\right)
$$

for $\left(x_{1}, x_{2}, x_{3}\right) \in \mathbb{R}^{n} \times \mathbb{R}^{n} \times \mathbb{R}^{n}$, for all $k=1, \ldots, N, i=$ $1, \ldots, N+1$ and for each $j=1, \ldots, m$.

For the more general case than one spatial dimension, an $N+1$ component system will be equivalent to the $N$ component system if

$$
f_{i}(x)=f_{k}(x), \quad g_{j, i}(x)=g_{j, k}(x)
$$

for $x \in \mathbb{R}^{n}$, for all $k=1, \ldots, N$ and for all $i=1, \ldots, N+1$ and for each $j=1, \ldots, m$ and there exists an ordering for each $\mathcal{W}_{i}$ such that $\hat{f}_{i}^{j}(x)=\hat{f}_{k}^{l}(x)$ for $j \in \mathcal{W}_{i}$ and $l \in \mathcal{W}_{k}$. The $N+1$ system is feedback equivalent to the $N$ system if

$$
\begin{gathered}
u_{j, i}\left(x_{i}(t), w_{l_{1}}^{i}\left(x_{l_{1}}(t)\right), w_{l_{2}}^{i}\left(x_{l_{2}}(t)\right), w_{l_{3}}^{i}\left(x_{l_{3}}(t)\right), \ldots\right)= \\
u_{j, k}\left(x_{k}(t), w_{m_{1}}^{k}\left(x_{m_{1}}(t)\right), w_{m_{2}}^{k}\left(x_{m_{2}}(t)\right), w_{m_{3}}^{k}\left(x_{m_{3}}(t)\right), \ldots\right)
\end{gathered}
$$

for $\left(x_{1}, x_{2}, x_{3}\right) \in \mathbb{R}^{n} \times \mathbb{R}^{n} \times \mathbb{R}^{n}$, for all $k=1, \ldots, N, i=$ $1, \ldots, N+1$, for each $j=1, \ldots, m$, for $l_{1}, l_{2}, \ldots, \in \mathcal{W}_{i}$ and for $m_{1}, m_{2}, \ldots, \in \mathcal{W}_{k}$.

Example 2 Returning to Example 1, the manner in which it was constructed was independent of the number of components, $N$. Furthermore, if we order

$$
\mathcal{W}_{i}=\{i-2, i-1, i+2 i+2\}
$$

for each $i$, the requirement of Equation 4 is satisfied. Hence, a system of size $N+1$ and a system of size $N$ defined by Example 1 are equivalent.

For notational convenience, we will stack all the states and vector fields from each component into one system description, $\dot{x}=f(x)+g(x) u(t)$ where

$x=\left[\begin{array}{c}x_{1} \\ x_{2} \\ \vdots \\ x_{N}\end{array}\right], u=\left[\begin{array}{c}u_{1} \\ u_{2} \\ \vdots \\ u_{N}\end{array}\right], f(x)=\left[\begin{array}{c}f_{1}\left(x_{1}\right) \\ f_{2}\left(x_{2}\right) \\ \vdots \\ f_{N}\left(x_{N}\right)\end{array}\right], g(x)=\left[\begin{array}{c}g_{1}\left(x_{1}\right) \\ g_{2}\left(x_{2}\right) \\ \vdots \\ g_{N}\left(x_{N}\right)\end{array}\right]$. 


\section{Stability OF Symmetric Systems}

This section presents the main theoretical result, which is that if a symmetric system is stable, then any larger system that is constructed from the original system is also stable. In a way, this is intuitively obvious, but this section formalizes and proves it. The following sections apply the result to a few of the well-known methods for formation control.

Proposition 1 Given a feedback symmetric system in one spatial dimension of size $N$, assume that it is stable in the sense of Lyapunov and furthermore that the Lyapunov function is of the form

$$
\begin{aligned}
& V(x)=\sum_{i=1}^{N} V_{i}\left(x_{i}, w_{i-1}^{+}\left(x_{i-1}\right), w_{i-1}^{-}\left(x_{i+1}\right)\right), \\
& \dot{V}(x)=\nabla V \cdot(f(x)+g(x) u(x)) \leq 0,
\end{aligned}
$$

and the $V_{i}$ are symmetric in the sense that

$$
\begin{aligned}
& V_{i}\left(x_{i}, w_{i-1}^{+}\left(x_{i-1}\right), w_{i+1}^{-}\left(x_{i+1}\right)=\right. \\
& \quad V_{j}\left(x_{j}, w_{j-1}^{+}\left(x_{j-1}\right), w_{j+1}^{-}\left(x_{j+1}\right) \quad \forall i, j=1, \ldots, N .\right.
\end{aligned}
$$

Then an equivalent feedback system of size $N+1$ is also stable in the sense of Lyapunov.

Proof: By direct computation,

$$
\begin{aligned}
\dot{V} & =\sum_{i=1}^{N} \sum_{j=1}^{N} \frac{\partial V_{i}}{\partial x_{j}}\left(f_{j}\left(x_{j}\right)+g_{j}\left(x_{j}\right) u\left(x_{j}, w_{j+1}^{-}\left(x_{j+1}\right), w_{j-1}^{+}\left(x_{j-1}\right)\right)\right) \\
& =\sum_{i=1}^{N} \sum_{j=i-1}^{i+1} \frac{\partial V_{i}}{\partial x_{j}}\left(f_{j}\left(x_{j}\right)+g_{j}\left(x_{j}\right) u\left(x_{j}, w_{j+1}^{-}\left(x_{j+1}\right), w_{j-1}^{+}\left(x_{j-1}\right)\right)\right) .
\end{aligned}
$$

By the assumption on the symmetry in $V$ and the fact that the system is symmetric,

$\dot{V}=N \sum_{j=N, 1,2} \frac{\partial V_{1}}{\partial x_{j}}\left(f_{j}\left(x_{j}\right)+g_{j}\left(x_{j}\right) u\left(x_{j}, w_{j+1}^{-}\left(x_{j+1}\right), w_{j-1}^{+}\left(x_{j-1}\right)\right)\right)$.

Since $\dot{V}(t) \leq 0$,

$$
\sum_{j=N, 1,2} \frac{\partial V_{1}}{\partial x_{j}}\left(f_{j}\left(x_{j}\right)+g_{j}\left(x_{j}\right) u\left(x_{j}, w_{j+1}^{-}\left(x_{j+1}\right), w_{j-1}^{+}\left(x_{j-1}\right)\right)\right) \leq 0 .
$$

By the same construction, for the $N+1$ equivalent system,

$\dot{V}=$

$(N+1) \sum_{j=N, 1,2} \frac{\partial V_{1}}{\partial x_{j}}\left(f_{j}\left(x_{j}\right)+g_{j}\left(x_{j}\right) u\left(x_{j}, w_{j+1}^{-}\left(x_{j+1}\right), w_{j-1}^{+}\left(x_{j-1}\right)\right)\right)$

which is less than or equal to zero by Equation 5 .

A theorem and proof for a more generally connected system follows by identical arguments, except the sum over the connected components is over the whole set of connected components, rather than just the two neighbors that are present in the one spatial dimension case. A theorem and proof for a time varying Lyapunov function also follows from the same arguments.

\section{EXAMPLES}

This section will complete Example 1 and present an additional example.

Example 3 Continuing Example 1, for a fleet of 5 agents, which has a strongly-connected topology, define a Lyapunov function as

$V=\frac{1}{2} \sum_{i=1}^{5}\left[\left(\dot{x}_{i}^{2}+\dot{y}_{i}^{2}\right)+\sum_{j}\left(\sqrt{\left(x_{i}-x_{j}\right)^{2}+\left(y_{i}-y_{j}\right)^{2}}-d_{i j}\right)^{2}\right]$,

where $j \in\{i-2, i-i, i+1, i+2\}$ and $d_{i j}$ is the desired distance between robots defined previously. By construction, this Lyapunov function satisfies the hypothesis of Proposition 1. Computing $\dot{V}$ gives

$$
\begin{aligned}
& \dot{V}=\nabla V \cdot(f+g u) \\
& =\sum_{i=1}^{5}\left[\begin{array}{c}
\sum_{j} 2\left(\frac{\sqrt{\left(x_{i}-x_{j}\right)^{2}+\left(y_{i}-y_{j}\right)^{2}}-d_{i j}}{\sqrt{\left(x_{i}-x_{j}\right)^{2}+\left(y_{i}-y_{j}\right)^{2}}}\left(x_{i}-x_{j}\right)\right) \\
\dot{x}_{i} \\
\sum_{j} 2\left(\frac{\sqrt{\left(x_{i}-x_{j}\right)^{2}+\left(y_{i}-y_{j}\right)^{2}}-d_{i j}}{\sqrt{\left(x_{i}-x_{j}\right)^{2}+\left(y_{i}-y_{j}\right)^{2}}}\left(y_{i}-y_{j}\right)\right) \\
\dot{y}_{i}
\end{array}\right] \\
& \cdot\left[\begin{array}{c}
\dot{x}_{i} \\
-\sum_{j} \frac{2\left(\sqrt{\left(x_{i}-x_{j}\right)^{2}+\left(y_{i}-y_{j}\right)^{2}}-d_{i j}\right)\left(x_{i}-x_{j}\right)}{\sqrt{\left(x_{i}-x_{j}\right)^{2}+\left(y_{i}-y_{j}\right)^{2}}}-k_{d} \dot{x}_{i} \\
-\sum_{j} \frac{2\left(\sqrt{\left(x_{i}-x_{j}\right)^{2}+\left(y_{i}-y_{j}\right)^{2}}-d_{i j}\right)\left(y_{i}-y_{j}\right)}{\sqrt{\left(x_{i}-x_{j}\right)^{2}+\left(y_{i}-y_{j}\right)^{2}}}-k_{d} \dot{y}_{i}
\end{array}\right] \\
& =\sum_{i=1}^{5}-k_{d}\left(\dot{x}_{i}^{2}+\dot{y}_{i}^{2}\right) \text {. }
\end{aligned}
$$

By Proposition 1, this will also hold for any $N$. Simulation results for a five-agent system are illustrated in Figures 3 and 4 with $k_{d}=0.25$. Figure 3 shows the trajectories for the individual agents, and Figure 4 shows the final configuration. Simulation results for a 17 -agent system are illustrated in Figures 5 and 6 with $k_{d}=0.5$. Figure 5 shows the trajectories for the individual agents, and Figure 6 shows the final configuration, illustrating stability of the system independent of the number of agents.

One must be careful in what can be concluded from $\dot{V} \leq 0$ in an example like this. LaSalle's theorem only applies in an invariant compact set, and since $V$ is invariant with respect to translations and rotations of a formation, it can not be directly applied in any global sense. A reader is referred to [13] for the details of the proof for local asymptotic stability to a formation. Furthermore, an additional complication addressed in [13] and somewhat in [2] is the issue of whether the formation is unambiguous. In the present example, the formation with five vehicles is unambiguous, but the larger one with 17 robots is not, although the simulation converged to the intended formation.

Example 4 This example considers formation control of a fleet of unicycle-like vehicles. Rather than being a distributed 


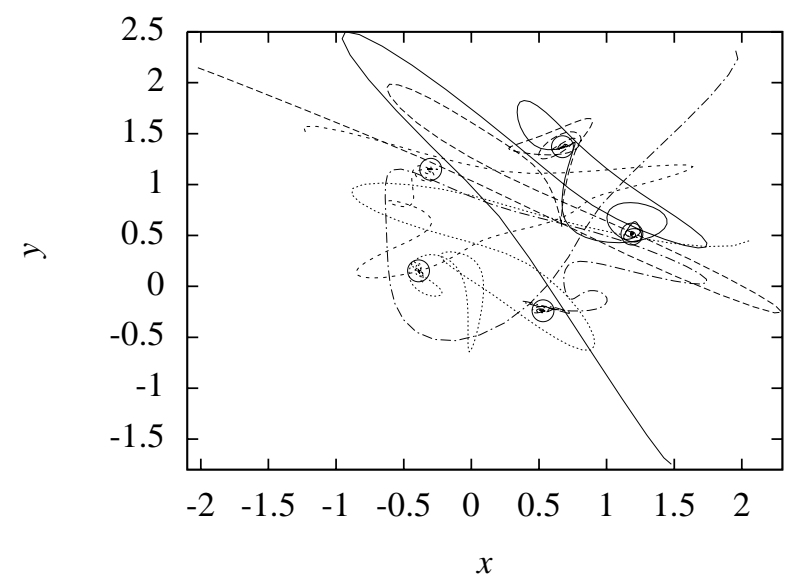

Fig. 3. Trajectories for distributed control for a five-vehicle system.

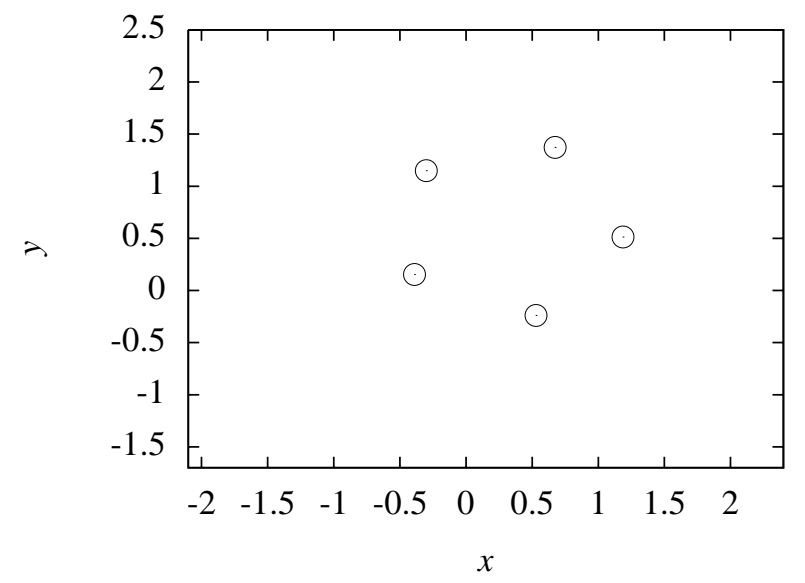

Fig. 4. Final formation for distributed control for a five-vehicle system.

algorithm, a global formation function is minimized. This example is motivated by the results in [12].

Each of the robots has dynamics given by

$$
\dot{x}_{i}=u_{1, i} \cos \theta_{i}, \quad \dot{y}_{i}=u_{1, i} \sin \theta_{i}, \quad \dot{\theta}_{i}=u_{2, i},
$$

where $u_{1, i}$ and $u_{2, i}$ are the inputs, which are the kinematic linear and angular velocities of the unicycle, respectively. It is well known that this model is dynamic feedback linearizable [4]. Defining

$$
\begin{aligned}
\dot{\xi}_{i} & =v_{1, i} \cos \theta+v_{2, i} \sin \theta \\
u_{1, i} & =\xi_{i} \\
u_{2, i} & =\frac{-v_{1, i} \sin \theta_{i}+v_{2, i} \cos \theta_{i}}{\xi_{i}}
\end{aligned}
$$

which gives the system

$$
\begin{aligned}
\dot{x}_{i} & =\xi_{i} \cos \theta_{i}, & \dot{\theta}_{i} & =\frac{1}{\xi_{i}}\left(-v_{1, i} \sin \theta_{i}+v_{2, i} \sin \theta_{i}\right), \\
\dot{y}_{i} & =\xi_{i} \sin \theta_{i}, & & \dot{\xi}_{i}=v_{1, i} \cos \theta_{i}+v_{2, i} \sin \theta_{i},
\end{aligned}
$$

which clearly has a singularity at $\xi_{i}=0$, which corresponds to zero velocity. If the desired trajectory is given

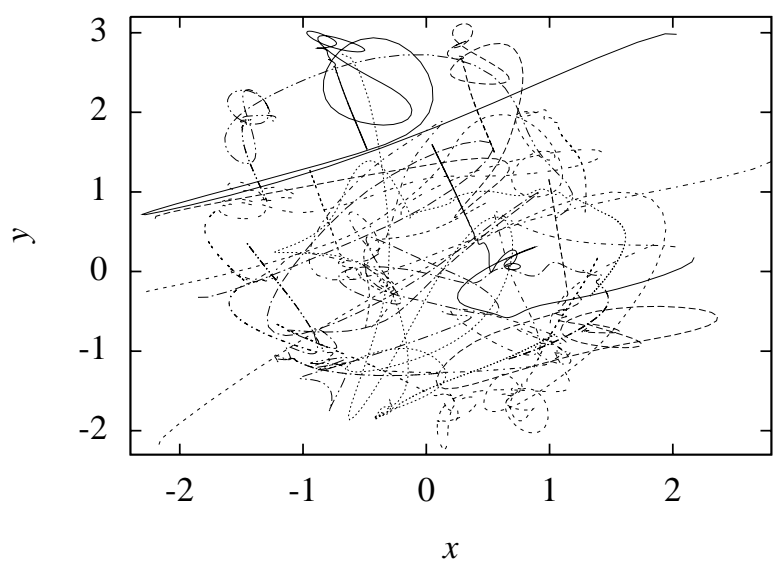

Fig. 5. Trajectories for distributed control for a 17-vehicle system.

by $\left(x_{i}^{d}(t), y_{i}^{d}(t)\right)$, then the inputs

$$
\begin{aligned}
& u_{1, i}=\ddot{x}_{i}^{d}-\left(x_{i}-x_{i}^{d}\right)-\left(\dot{x}_{i}-\dot{x}_{i}^{d}\right) \\
& u_{2, i}=\ddot{y}_{i}^{d}-\left(y_{i}-y_{i}^{d}\right)-\left(\dot{y}_{i}-\dot{y}_{i}^{d}\right)
\end{aligned}
$$

achieve asymptotic tracking. To see this, define

$$
e_{x, i}=x_{i}-x_{i}^{d}, \quad e_{y, i}=y_{i}-y_{i}^{d}
$$

from which the error dynamics using those inputs are

$$
\frac{d}{d t}\left[\begin{array}{c}
e_{x, i} \\
\dot{e}_{x, i} \\
e_{y, i} \\
\dot{e}_{y, i}
\end{array}\right]=\left[\begin{array}{c}
\dot{e}_{x, i} \\
-e_{x, i}-\dot{e}_{x, i} \\
\dot{e}_{y, i} \\
-e_{y, i}-\dot{e}_{y, i}
\end{array}\right] .
$$

Defining $V_{i}=1 / 2\left(e_{x, i}^{2}+\dot{e}_{x, i}^{2}+e_{y, i}^{2}+\dot{e}_{y, i}^{2}\right)$ gives

$$
\dot{V}_{i}=\left[\begin{array}{c}
e_{x, i} \\
\dot{e}_{x, i} \\
e_{y, i} \\
\dot{e}_{y, i}
\end{array}\right] \cdot\left[\begin{array}{c}
\dot{e}_{x, i} \\
-e_{x, i}-\dot{e}_{x, i} \\
\dot{e}_{y, i} \\
-e_{y, i}-\dot{e}_{y, i}
\end{array}\right]=-\dot{e}_{x, i}^{2}-\dot{e}_{y, i}^{2} .
$$

Since $V$ is positive definite, radially unbounded and continuously differentiable, from LaSalle's invariance principle we can conclude global asymptotic stability.

So, for this system the global Lyapunov function $V=$ $\sum_{i=1}^{N} V_{i}$ can be defined, and by Proposition $1, \dot{V} \leq 0$ for all $N$ since it was true for $N=1$. Simulation results are illustrated in Figures 7 and 8 for six and 13 unicycles respectively. In each case the desired trajectory is given by $x_{i}^{d}=t$ and $y_{i}^{d}=\sin (t)+i-\frac{N}{2}$. Each $x$ mark on the figures represent a specific times, which illustrate that not only do the robots track the desired trajectories in space, they also are doing so at the desired time.

As with the previous example, one must take care to draw the proper conclusions from $\dot{V} \leq 0$. The main results in [12] were, similar to this paper, a means to combine individual Lyapunov functions in a useful manner. In particular, that paper presented definition of a formation function and bounds on the formation speed that ensure stability of the system to the desired formation. In such a case, the 


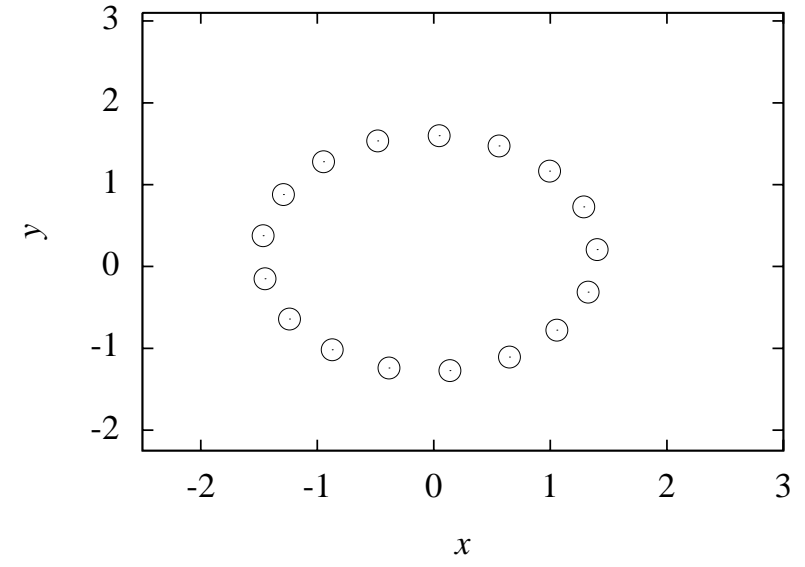

Fig. 6. Final formation for distributed control for a 17-vehicle system.

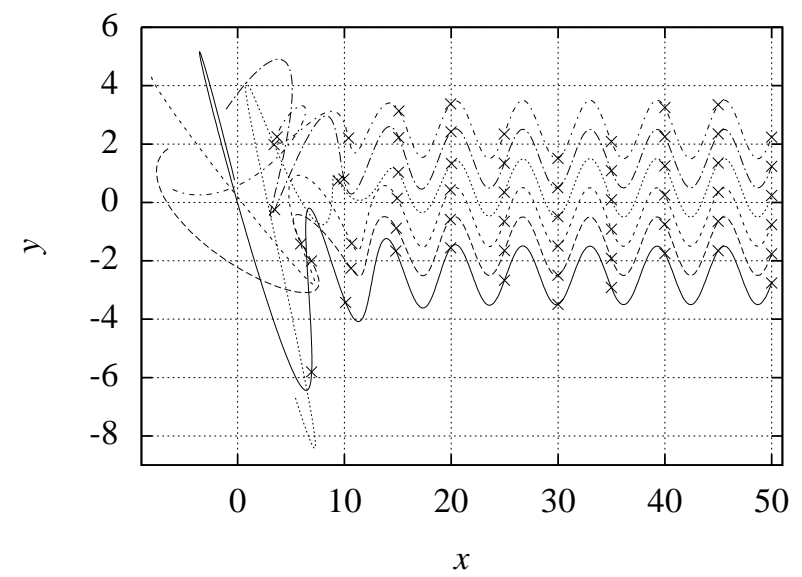

Fig. 7. Trajectories for six unicycle robots.

proper interpretation from the Lyapunov function requires consideration of the additional requirements for Lyapunov stability of nonautonomous systems which requires, among other things, for the Lyapunov function to be bounded above and below by class $\mathcal{K}$ functions, a stricter bound on $\dot{V}$, etc. $\square$

\section{Conclusions}

This paper considered the stability of coordinated and distributed systems, with an application focus on coordinated control of systems of mobile robots. Unlike our prior work, it formulated the problem as a nonlinear extension of the work in [1], [14], which was directed toward spatially periodic systems "built-up" from periodically interconnected components. Observing that many of the formation control algorithms in the literature are not limited by the number of components, but often are limited by assuming specific dynamics, the main contribution was to formulate an theoretical framework in which stability of many distributed systems can be considered. The result was demonstrated on two systems, one of which was fully distributed and the other of which was no decentralized.

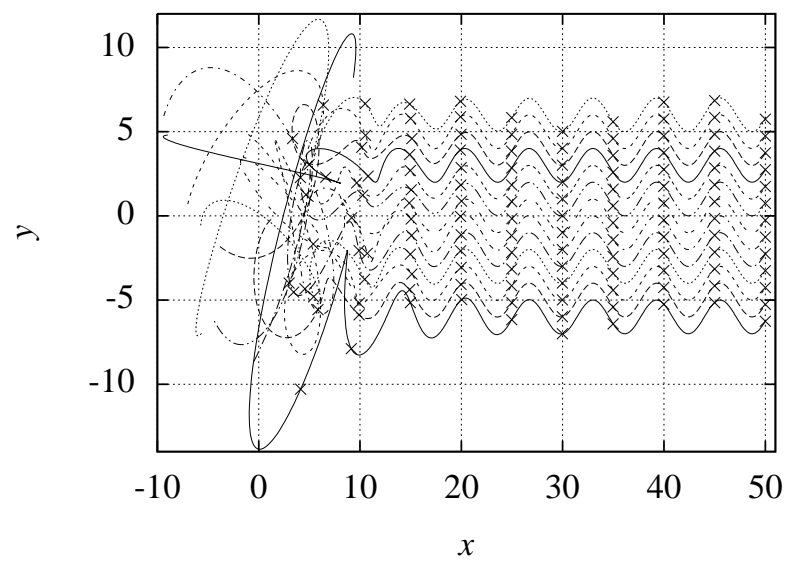

Fig. 8. Trajectories for 13 unicycle robots.

\section{REFERENCES}

[1] Raffaello DÁndrea dna Geir E. Eullerud. Distributed control design for spatially interconnected systems. IEEE Transactions on Automatic Control, 48(9):1478-1495, September 2003.

[2] J. Alexander Fax and Richard M. Murray. Information flow and cooperative control of vehicle formations. IEEE Transactions on Automatic Control, 49(9):1465- 1476, 2004.

[3] N.E. Leonard and E. Fiorelli. Virtual leaders, artificial potentials, and coordinated cont. pages 2968-2973, 2001.

[4] A. De Luca, G. Oriolo, and M. Vendittelli. Stabilization of the unicycle via dynamic feedback linearization. In 6th IFAC Symposium on Robot Control, pages 397-402, 2000.

[5] M. Brett McMickell. Reduction and Control of Nonlinear Symmetric Distributed Robotic Systems. PhD thesis, University of Notre Dame, 2003.

[6] M. Brett McMickell and Bill Goodwine. Reductionand controllability of symmetric distributed systems with robotic applications. In International Conference on Intelligent Robotics and Systems, volume 3 , pages 1232-1236. IEEE/RSJ, 2001.

[7] M. Brett McMickell and Bill Goodwine. Reduction and controllability of robotic systems with drift. In Proceedings of the 2002 IEEE International Conference on Robotics and Automation, 2002.

[8] M. Brett McMickell and Bill Goodwine. Motion planning for symmetric distributed robotic systems. In 2003 IEEE International Conference on Robotics and Automation, 2003.

[9] M. Brett McMickell and Bill Goodwine. Reduction and nonlinear controllability of symmetric distributed systems. International Journal of Control, 76(18):1809-1822, 2003.

[10] M. Brett McMickell and Bill Goodwine. Motion planning for nonlinear symmetric distributeed robotic systems. International Journal of Robotics Research, 26(10):1025-1041, October 2007.

[11] M. Brett McMickell, Bill Goodwine, and Luis Antonio Montestruque. Micabot: A robotic platform for large-scale distributed robotics. In 2003 IEEE International Conference on Robotics and Automation, 2003.

[12] Petter Ögren, Magnus Egerstedt, and Xiaoming Hu. A control lyapunov function approach to multiagent coordination. IEEE Transactions on Robotics and Automation, 18(5):847-851, October 2002.

[13] Reza Olfati-Saber and Richard M. Murray. Distributed cooperative control of multiple vehicle formations using structural potential functions. In Proceedings of the 2002 IFAC World Congress.

[14] Benjamin Recht and Raffaello DÁndrea. Distributed control of systems over discrete groups. IEEE Transactions on Automatic Control, 49(9):1446-1452, September 2004.

[15] Elon Rimon and Daniel E. Koditschek. Exact robot navigation using artificial potential functions. IEEE Transactions on Robotics and Automation, 8(5):501-518, 1992. 